International Institute for Applied Systems Analysis - A-2361 Laxenburg - Austria Tel: +432236 807 • Fax: +43223671313 • E-mail: info@iiasa.ac.at • Web: www.iiasa.ac.at

INTERIM REPORT IR-98-064/August

\title{
Rank Order for a Rehabilitation Program Using Multiple Criteria
}

Grazia Concilio(pndn1001@mlx.pandora.it)

Pekka Korhonen(korhonen@iiasa.ac.at)

Marga reta So ismaa(soismaa@iiasa.ac.at)

Approved by

Gordon Mac Donald(mac don@ilasa.ac .at)

Director, IIASA

Interim Reports on work of the International Institute for Applied Systems Analysis receive only limited review. Views or opinions expressed herein do not necessarily represent those of the Institute, its National Member Organizations, or other organizations supporting the work. 


\section{Contents}

1. Introduction 1

2. Theoretical Foundation of the "Minimum Violation" Principle 2

3. Description of the Matera rehabilitation program (Agostiano [1995]) 5

$\begin{array}{ll}3.1 \text { The evaluation matrix } & 6\end{array}$

$\begin{array}{ll}3.2 \text { Review of previous approaches } & 6\end{array}$

3.3 Results from Agostiano [1995] 8

3.4 Critique of Agostiano's [1995] results 11

4. Rank Order Using Minimum Violation Principle 13

4.1 Cardinal information 14

4.2 Ordinal information 15

$\begin{array}{ll}\text { 5. Concluding remarks } & 17\end{array}$ 


\section{Abstract}

In this study we investigate an urban planning problem where an area is to be rehabilitated. The area is divided into several sub-areas any of which could be the starting point for the rehabilitation process. The ultimate goal is to find a rank order for the alternative subareas, which simultaneously solves the problem of where to start the rehabilitation. If all information were given on the ordinal scale, we could use e.g. the classical minimum violation principle to solve this problem. In this paper, we have generalized this approach to cover the cardinal scale and pairwise information.

Keywords: Urban Planning, Rehabilitation, Multiple Criteria Ranking 


\section{Acknowledgments}

Pekka Korhonen's research was supported, in part, by grants from the Academy of Finland.

Grazia Concilio's research was supported by "Programma di Scambi Internazionali tra L'Università degli Studi di Napoli Federico II ed Istituti di Ricerca Stranieri per la mobilità di breve durata di docenti e ricercatori". 


\section{About the Authors}

Grazia Concilio comes from the University of Bari, Italy. She had a 4 month assignment with the DAS project during the period January-April 1998.

Pekka Korhonen is Project Leader of the Decision Analysis and Support Project at IIASA, and also Professor of Statistics at the Department of Economics and Management Science, Helsinki School of Economics and Business Administration.

Margareta Soismaa was a Research Scholar with the DAS project during the period July 1997 through August 1998. She has since returned to her home university, the Helsinki School of Economics and Business Administration, where she is a Senior Researcher at the Doctoral Program Center. 


\title{
Rank Order for a Rehabilitation Program Using Multiple Criteria
}

\author{
Grazia Concilio \\ Pekka Korhonen \\ Margareta Soismaa
}

\section{Introduction}

Issues dealing with urban planning, conservation, and rehabilitation are not only economically and politically relevant but also analytically interesting. They include traditional quantifiable aspects such as costs and benefits but, additionally, they also involve intangibles, incommensurable effects, and qualitative features. In particular, when evaluating plans or projects in a historical area there is a need to integrate economic, social, architectural, and historical aspects of city life and to take these into account simultaneously. In other words, these problems exhibit all the characteristics of Multiple Criteria Evaluation Problems.

Multiple criteria analysis was recently introduced in the domain of urban planning (see, e.g., Nijkamp [1975], Delft and Nijkamp [1977], Rietveld [1980], Voogd [1982], [1983]). It rapidly showed its potential for conservation/rehabilitation applications, particularly those oriented towards the evaluation of plans or projects (Fusco Girard [1987], Nijkamp [1988], Albers and Nijkamp [1989]). In these cases, the evaluation process which considers several criteria can be used to support the decision maker to choose between different alternatives in two ways. It can either provide a rank order of the alternative plans or projects (Albers [1989], Giordano [1997], De Toro [1997], Bizzarro and Ferretti [1997]) or it can provide methodological assistance to help the decision maker select his/her most preferred or the best alternative.

The use of multiple criteria analysis has two definite advantages in the domain of urban planning, and specifically in the domain of urban conservation/rehabilitation. First, due to the need of strong formalization, it calls for a thorough examination of the decision environment and, thus, enables a deep understanding of it. Secondly, it allows the inclusion of different points of view, which, in this domain, is otherwise a difficult task to carry out.

We study a problem of urban rehabilitation, which involves five sub-areas, each of which represents a rehabilitation project. The sub-areas are to be ranked according to three criteria that are divided into seventeen sub-criteria in all. The rank order then determines where to start the rehabilitation process. Agostiano [1995] analyzed the same problem using five different methods. But the results are very conflicting and it is 
difficult to explain those conflicting rankings on the basis of the theoretical foundations of the methods.

In this paper we propose a ranking principle based on the "Minimum Violation" principle. The best rank order is searched for by trying to minimize the number of conflicting pairwise orders with those defined by the original criteria. The principle is also generalized to cover the cardinal scale and pairwise information. The results of this approach are compared to those of Agostiano [1995]. The differences are considered in light of minimum violation principle.

The paper proceeds as follows. In Chapter 2 we present the theoretical foundations of the minimum violation principle. In Chapter 3 we introduce in detail the ranking problem of the Matera rehabilitation program. We also review the previous approaches used by Agostiano [1995], present the respective results, and criticize them. In Chapter 4 we present our results for both cardinal and ordinal data. We end the paper with concluding remarks in Chapter 5.

\section{Theoretical Foundation of the "Minimum Violation" Principle}

We assume that a single decision maker $(D M)$ has to rank $n(n>0)$ explicitly defined deterministic decision alternatives using $p(p>1)$ different criteria. The basic data set can be given in an $n \times p$ matrix $A \in \mathfrak{R}^{\mathrm{n} \times \mathrm{p}}$ whose elements $a_{i j}, i \in I=\{1,2, \ldots, n\}$ and $j \in J=$ $\{1,2, \ldots, p\}$ represent the criterion (numerical) values on alternatives. We use notation $A_{i}$ or simply index $i$ to refer to the alternative in row $i$. Correspondingly, we use notation $a_{j}$ or simply index $j$ to refer to the criterion in column $j$. In reference to the $j^{\text {th }}$ criterion vector, we use $\mathbf{a}_{\mathrm{j}}$. Furthermore, we denote $\mathbf{1}=[1, \ldots, 1]^{\mathrm{T}}$.

We further assume that each criterion is to be maximized. To each criterion, we associate a function $v_{\mathrm{j}}:\left\{\mathrm{a}_{1 \mathrm{j}}, \mathrm{a}_{2 \mathrm{j}}, \ldots, \mathrm{a}_{\mathrm{nj}}\right\} \rightarrow \mathfrak{R}, \mathrm{j}=1,2, \ldots, \mathrm{p}$, which defines the preference structure of a $\mathrm{DM}$ over the alternatives. The resulting matrix is called a value matrix and denoted by $\mathrm{V}$ with elements $v_{i j}$, $i \in I$ and $j \in J$. We say that the value structure is given on a cardinal scale if the scale is at least an interval one. In addition, in case the value scale is cardinal, we assume that it is a linear transformation of the original one. The function is assumed to be strictly increasing.

Our problem is now to find an arrangement of the form $A_{i} \prec A_{j} \prec \ldots \prec A_{k}$, where " $\prec$ " is the symbol for "less preferred to", and the subscripts i, $\mathrm{j}, \ldots, \mathrm{k}$ range over mutually exclusive and exhaustive subsets of integers $1,2, \ldots, \mathrm{n}$. The alternatives can be ranked according to each criterion, but there is no unique "optimal" rank for the alternatives in the presence of multiple criteria. Actually, there is not even a unique principle to measure the similarity of two rankings.

As a dissimilarity measure of two rankings, we will use a pairwise violation principle: the proportional number of conflicting pairwise orders in two rankings. In case we are able to use cardinal information, we may take into account not only conflicting orders, but also the differences of the values of the criteria in the pairs, which are in a conflicting order. This kind of measure is easy to handle, if we present the original data by means of a pairwise comparison matrix.

Definition 1. The $n \times n$ matrix $C\left(a_{j}\right)$ is an unweighted pairwise comparison matrix describing the rank order of observations according to variable $a_{i}$ if 


$$
\begin{aligned}
& \mathrm{C}_{\mathrm{hk}}\left(\mathrm{a}_{\mathrm{j}}\right)= \begin{cases}1, & \text { if } \mathrm{a}_{\mathrm{hj}}>\mathrm{a}_{\mathrm{kj}} \\
0, & \text { if } \mathrm{a}_{\mathrm{hj}}=\mathrm{a}_{\mathrm{kj}} \\
-1, & \text { if } \mathrm{a}_{\mathrm{hj}}<\mathrm{a}_{\mathrm{kj}}\end{cases} \\
& \mathrm{C}_{\mathrm{hh}}\left(\mathrm{a}_{\mathrm{j}}\right)=0 \\
& \forall \mathrm{h}, \mathrm{k}=1,2, \ldots, \mathrm{n} .
\end{aligned}
$$

Definition 2. The $\mathrm{n} \times \mathrm{n}$ matrix $\mathrm{C}\left(\mathrm{a}_{\mathrm{j}}\right)$ is a weighted pairwise comparison matrix describing the rank order of observations according to variable $\mathrm{a}_{\mathrm{j}}$ if

$$
\begin{aligned}
& \mathrm{C}_{\mathrm{hk}}\left(\mathrm{a}_{\mathrm{j}}\right)=\quad \mathrm{a}_{\mathrm{hj}}-\mathrm{a}_{\mathrm{kj}} \\
& \forall \mathrm{h}, \mathrm{k}=1,2, \ldots, \mathrm{n} .
\end{aligned}
$$

For brevity, we replace notation $\mathrm{C}\left(\mathrm{a}_{\mathrm{j}}\right)$ by $\mathrm{C}^{\mathrm{j}}$ and $\mathrm{C}_{\mathrm{hk}}\left(\mathrm{a}_{\mathrm{j}}\right)$ by $\mathrm{c}_{\mathrm{hk}}^{\mathrm{j}}$, respectively. Moreover, we denote

$$
\mathbf{f}_{j}=\left[c_{11}^{j}, c_{12}^{j}, \ldots, c_{1 n}^{j}, \ldots, c_{n 1}^{j}, \ldots, c_{n n}^{j}\right]^{T} \text {. }
$$

Note that $\mathbf{1}^{\mathrm{T}} \mathbf{f}_{\mathrm{j}}=\sum_{\mathrm{h}=1}^{\mathrm{n}} \sum_{\mathrm{m}=1}^{\mathrm{n}} \mathrm{c}_{\mathrm{hm}}^{\mathrm{j}}=0, \forall \mathrm{j}=1,2, \ldots, \mathrm{p}$.

Now we will define the following similarity measure for two rankings:

$$
\tau_{\mathrm{ij}}=\frac{\mathbf{f}_{\mathrm{i}}^{\mathrm{T}} \mathbf{f}_{\mathrm{j}}}{\sqrt{\mathbf{f}_{\mathrm{i}}^{\mathrm{T}} \mathbf{f}_{\mathrm{i}} \sqrt{\mathbf{f}_{\mathrm{j}}^{\mathrm{T}} \mathbf{f}_{\mathrm{j}}}}} .
$$

Originally Daniels [1943-46] showed that $\tau_{\mathrm{ij}}$ is Kendall's rank correlation coefficient, when the definition of $\mathbf{f}_{\mathrm{i}}$ and $\mathbf{f}_{\mathrm{j}}$ is based on an unweighted pairwise matrix $\mathrm{C}^{\mathrm{i}}$ and $\mathrm{C}^{\mathrm{j}}$. Furthermore, Korhonen [1986] showed that $\tau_{\mathrm{ij}}$ is an ordinary Pearson product-moment correlation coefficient between criteria $a_{i}$ and $a_{j}$, when the definition of both $\mathbf{f}_{i}$ and $\mathbf{f}_{j}$ is based on weighted pairwise matrices. Thus, it is very natural to generalize this definition also to the case where one of the criteria $\mathrm{i}$ or $\mathrm{j}$ is based on an unweighted pairwise matrix and the other one on a weighted matrix. Hence, we have a measure for conflicting rankings. The measure is based on the (weighted or unweighted) proportion of pairwise orders in conflict. It is scaled for the interval $[-1,1]$. In case $\tau_{\mathrm{ij}}=1$, the rank orders are exactly the same, and in case $\tau_{\mathrm{ij}}=-1$, they are totally opposite. In the sequel, this measure is simply called a correlation coefficient without any additional specifications.

Let us now consider our estimation problem. In other words, we have to find the "best" pairwise comparison matrix $\mathrm{C}(\mathrm{y})$, which defines a complete rank order for the alternatives. Because no ties are allowed, $c_{h k}(y) \neq 0$, if $h \neq k$. Equation (2.1) is not, however, a sufficient condition to define a transitive ordering for alternatives. Bowman and Colantoni [1972] 
proved that the ordering is transitive if it is transitive for every triple of alternatives. Therefore, we can guarantee the existence of a transitive ordering for all alternatives by making every triple transitive. This condition can be set as follows (see, Bowman and Colantoni [1972]):

$$
c_{h i}(y)+c_{i j}(y)+c_{j h}(y) \leq 1, \text { for all h, i, j }=1,2, \ldots, n \text {. }
$$

Now we can summarize the sufficient conditions, which guarantees that the matrix $\mathrm{C}(\mathrm{y})$ defines a complete ordering for alternatives $A_{i}, i=1,2, \ldots, n$. For convenience, we denote by $\mathrm{U}$ the unknown matrix $\mathrm{C}(\mathrm{y})$ we try to solve.

$$
\left\{\begin{array}{lll}
\mathrm{u}_{\mathrm{ij}} & =-1,1 & , \mathrm{i} \neq \mathrm{j} \\
\mathrm{u}_{\mathrm{ij}}+\mathrm{u}_{\mathrm{ji}} & =0 & , \text { for all } \mathrm{i}, \mathrm{j}=1,2, \ldots, \mathrm{n} \\
\mathrm{u}_{\mathrm{ij}}+\mathrm{u}_{\mathrm{jh}}+\mathrm{u}_{\mathrm{hi}} \leq 1 & , \text { for all } \mathrm{i}, \mathrm{j}, \mathrm{h}=1,2, \ldots, \mathrm{n} \\
\mathrm{u}_{\mathrm{ii}} & =0 & , \text { for all } \mathrm{i}=1,2, \ldots, \mathrm{n}
\end{array}\right.
$$

Let's now define the vector standing for the unknown ranking:

$$
\gamma=\left[\mathrm{u}_{11}, \mathrm{u}_{12}, \ldots, \mathrm{u}_{1 \mathrm{n}}, \ldots, \mathrm{u}_{\mathrm{n} 1}, \ldots, \mathrm{u}_{\mathrm{nn}}\right]^{\mathrm{T}}
$$

Because $\gamma^{\mathrm{T}} \gamma=\mathrm{n}(\mathrm{n}-1)$, then the correlation coefficient between the unknown ranking $\gamma$ and the variable $\mathrm{j}$ is as follows:

$$
\rho_{\mathrm{j}}=\frac{1}{\mathrm{n}(\mathrm{n}-1)} \frac{\gamma^{\mathrm{T}} \mathbf{f}_{\mathrm{j}}}{\sqrt{\mathbf{f}_{\mathrm{j}}^{\mathrm{T}} \mathbf{f}_{\mathrm{j}}}}
$$

To find the best ranking can be formulated as the following multiple criterion problem:

$$
\max \rho_{\mathrm{j}}=\frac{1}{\mathrm{n}(\mathrm{n}-1)} \frac{\gamma^{\mathrm{T}} \mathbf{f}_{\mathrm{j}}}{\sqrt{\mathbf{f}_{\mathrm{j}}^{\mathrm{T}} \mathbf{f}_{\mathrm{j}}}}=\mathrm{A} \sum_{\mathrm{h}=1}^{\mathrm{n}} \sum_{\mathrm{m}=1}^{\mathrm{n}} \mathrm{u}_{\mathrm{hm}} \mathrm{c}_{\mathrm{hm}}^{\mathrm{j}}, \mathrm{j}=1,2, \ldots, \mathrm{p}
$$

subject to constraints (2.6a-d),

$$
\text { where } A=\frac{1}{n(n-1) \backslash \sqrt{\sum_{h=1}^{n} \sum_{m=1}^{n}\left(c_{h m}^{j}\right)^{2}}} \text {. }
$$


Problem (2.8) is a typical multiple criteria problem having no unique solution. A "reasonable" solution is called nondominated and defined as follows:

Definition 3. A rank order $\mathbf{u}^{*}, \mathbf{u}^{*} \in V$, is nondominated iff $\nexists \mathbf{u}, \mathbf{u} \in V$, such that $\rho^{*}$ $\geq \rho$ and $\rho^{*} \neq \rho$, where $\rho^{*}$ is standing for the correlation coefficients corresponding to $\mathbf{u}^{*}$ and $\rho$ is standing for the correlation coefficients corresponding to $\mathbf{u}$.

Definition 4. An alternative $\mathbf{u}^{*}, \mathbf{u}^{*} \in \mathrm{V}$, is weakly-nondominated iff $\nexists \mathbf{u}, \mathbf{u} \in \mathrm{V}$, such that $\rho^{*}>\rho$, where $\rho^{*}$ is standing for the correlation coefficients corresponding to $\mathbf{u}^{*}$ and $\rho$ is standing for the correlation coefficients corresponding to $\mathbf{u}$.

If a rank order is not nondominated it is dominated.

Obviously, there are many nondominated rankings for each problem. Because no single ranking exists with the maximum values on all criteria, the rational DM will choose one nondominated ranking. Which alternative is chosen depends on the DM's preference structure. To be able to make a decision, he/she needs a tool to help him/her to find his/her "best" ranking. In multiple criteria decision making (MCDM), the most preferred is usually used as the synonym for the best. Generally, it is not an easy task to develop a method that helps a DM to find the most preferred ranking by means of model (2.8). With reference to one approach, see Korhonen and Soismaa [1981].

However, in our approach we enumerate all possibilities and then use a discrete multiple criteria method to solve the problem. Being biased, we use our own system VIMDA (A Visual Multiple Criteria Decision Support System for Discrete Alternatives with Numerical Data), which is based on Discrete Reference Direction Method developed by Korhonen [1988].

\section{Description of the Matera rehabilitation program (Agostiano [1995])}

The problem area under investigation is the "Sassi" neighborhood in Matera, a town in southern Italy, situated on the right hand side of a deep gorge. The first settlements date back to the Paleolithic age. It is also possible to find evidence from Hellenic and Roman ages. The first settlements were basically in caves and only later in the 7th and 8th centuries man-made dwellings were added to the natural caves giving the "Sassi" a rare mixture of natural and built living environment. This old part of the town is characterized by different small courts with their own small squares, steps, buildings, and caves, which are organized on different levels because of the morphology of the place.

The actual condition of the "Sassi" is the object of several scientific investigations and of many special regional and national laws. Several plans have been developed but only a recent law passed in 1986 gives enough power to the local government and enables the use of those plans already developed. The first executive plan refers to half of the total "Sassi" area. It divides the area into 18 sub-areas, which are differentiated by dimensions, future activities, and intervention stakeholders. Each sub-area requires a unique executive design and has been divided into minimal units of intervention, i.e. those parts of the sub-area that require simultaneous intervention. 
This study deals with the 5 most promising sub-areas (numbers 7, 8, 9, 10, and 12) of the 18 sub-areas in the first executive plan. The objective is to find a rank order for the 5 sub-areas, which at the same time determines where to start the rehabilitation.

\subsection{The evaluation matrix}

Each sub-area is evaluated with three main criteria. They deal with economic and technical feasibility, historical and environmental aspects, and social objectives. Each criterion is divided into sub-criteria that can be described in numbers. Economic and technical feasibility (criterion A) is divided into 7 sub-criteria. Historical and environmental aspects (criterion B) comprise 6 sub-criteria. Social objectives (criterion C) contain 4 sub-criteria. Thus, the data consists of 17 sub-criteria in all. Table 1 describes the evaluation matrix.

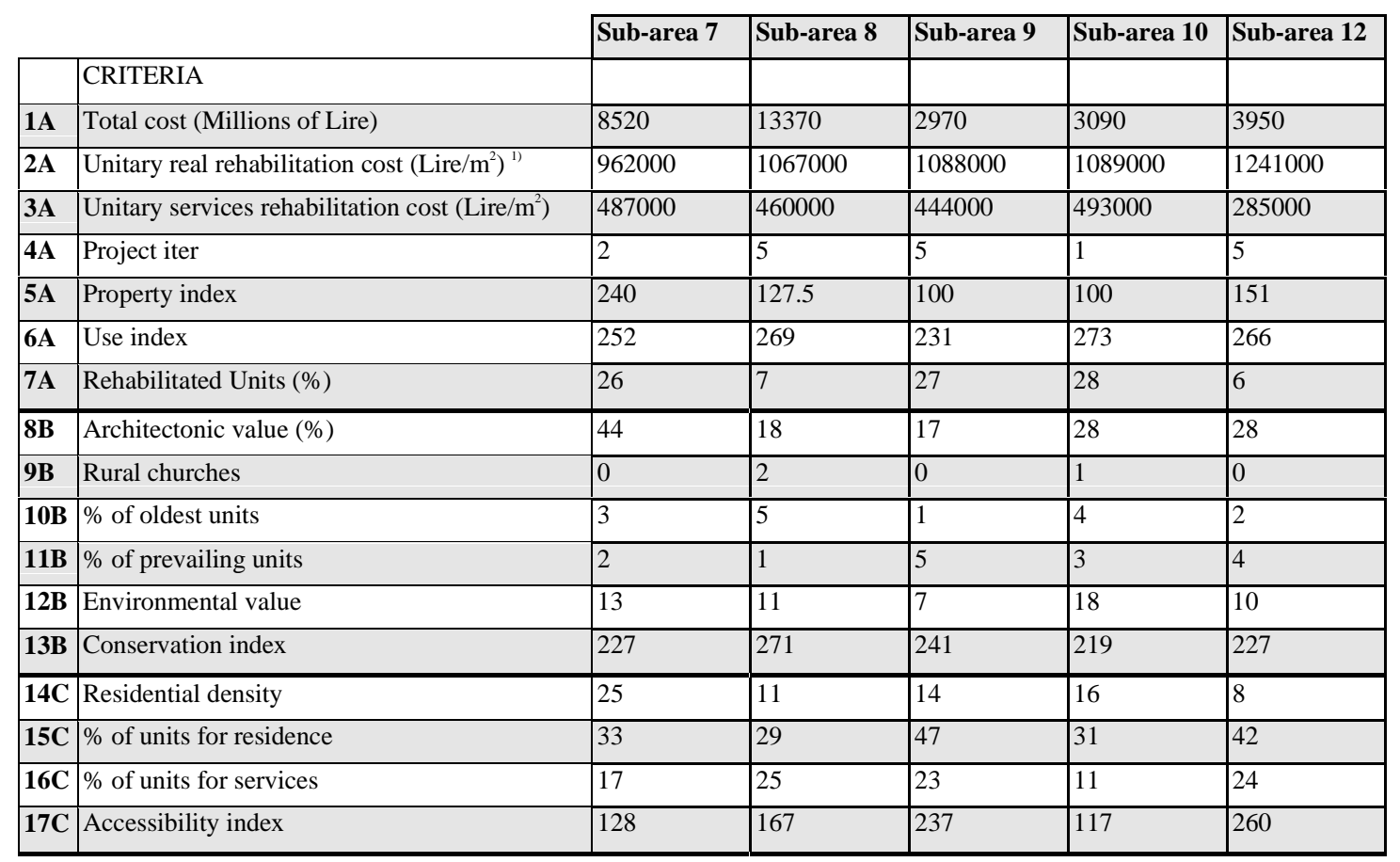

Table 1. The evaluation matrix.

As can be seen from Table 1, criteria 4A, 10B, and 11B are given on ordinal scales, whereas the rest are given on cardinal ones. In the sequel, for brevity, we call a criterion on an ordinal (cardinal) scale an ordinal (cardinal) criterion.

\subsection{Review of previous approaches}

Agostiano [1995] applied five different multiple criteria methods in order to find the rank order for the five sub-areas on the basis of the 17 sub-criteria. The following methods were used: (i) forecasted value, (ii) concordance analysis, (iii) numerical

\footnotetext{
1) Agostiano [1995] gave sub-areas 9 and 10 the same ranking on this sub-criterion.
} 
interpretation, (iv) regime analysis according to Hinloopen-Nijkamp, and (v) score method according to Israels-Keller (for the methods see e.g. Nijkamp et al. [1990] and Voogd[1983]). Although some of these methods allow the use of cardinal information, Agostiano [1995] used only ordinal data by transforming the original evaluation matrix into the respective ranking matrix.

The method of forecasted value uses either ordinal or cardinal information. In the latter case it is known as weighted sum. Starting from the evaluation matrix, Agostiano [1995] first transforms all criterion values into ordinal numbers. The "forecasted value" of each alternative is then computed by multiplying the value of each sub-criterion by its respective weight and adding the products. The alternatives are ranked according to the scores and the one achieving the highest one is considered the best.

Also concordance analysis (see Roy [1968, 1972]) can make use of either ordinal or cardinal information. Here ordinal data was used. The method, however, requires cardinal information on weights. For each pair of alternatives, the concordance set is first determined; i.e., the set of all criteria corresponding to which the first alternative is at least preferable to the second one. The concordance index is then computed to indicate the weight of the concordance set. It is the sum of the standardized weights of the criteria belonging to the respective concordance set. Next the sum of the concordance indices is computed for each alternative. The one with the highest score is considered the best one.

The method of numerical interpretation uses qualitative information. Each pair of alternatives is compared to each pair of criteria. For each pair of alternatives a square matrix (where the number of criteria determines the dimension) is computed. The cells in the matrix $(+1,0$, or -1$)$ are determined based on the rank orders of the pairs of criteria and the respective weights. The information in these matrices is then aggregated by summing the upper triangular parts of each matrix into a cell in a new matrix where the number of alternatives determines the dimension. Finally a score is computed for each alternative by calculating row sums of the square matrix. The alternative with the highest score is regarded as the most favorable one.

To some extent regime analysis according to Hinloopen-Nijkamp (Hinloopen et al. [1983]) can be interpreted as an ordinal generalization of pairwise comparison methods such as concordance analysis (Nijkamp et al. [1990]). The ordinal evaluation matrix and the possibility to use ordinal weights constitute the difference between these two approaches. The focus of regime analysis according to Hinloopen-Nijkamp is on the sign of the rank orders between two alternatives. A regime matrix that contains regime vectors for each pair of alternatives is first computed. The elements of the vectors are $(+1,-1$, or 0$)$ depending on whether the sign of differences between the rank orders of the criteria are positive, negative, or zero. For each pair of alternatives we then calculate a weighted sum over the criteria and get an index (a sum), the sign of which determines whether one alternative is preferred to another one. Because of ordinal weight information the sign of the index is not always unambiguous. To circumvent this difficulty assuming a uniform probability distribution the set of feasible weights is partitioned so that for each subset of weights a definite conclusion can be drawn about the sign of the index. The final ranking is determined by computing for each alternative a success index by adding the probabilities of this alternative being preferred to the other ones and dividing the sum by the number of alternatives minus one. The alternative corresponding to the highest success index is considered to be the best one. 
Score method according to Israels-Keller (Israels and Keller [1986]) is related to regime analysis. In this method all the quantitative weight alternatives generated by the qualitative weight vector are taken into account. These weight vectors are applied consecutively to the scores of the evaluation matrix, in this case those of the ranking matrix. For each alternative the result is a score, a weighted sum valid for a certain set of weights. The higher the score compared to the other alternatives the more attractive that alternative is. The scores are then used to rank the alternatives. The one with the highest score is regarded as the most preferred one.

It should be pointed out that Agostiano [1995] used the methods in two phases. The sub-areas were first ranked within each of the three criteria, that is, by using the respective sub-criteria. These three rank orders were then used as data in the second phase.

\subsection{Results from Agostiano [1995]}

Using the five methods from above Agostiano [1995] carried out the analyses by employing four different scenarios where the importance of the main criteria varies. In scenario 1 all criteria are considered to be of equal importance. Criterion $\mathrm{A}$ is regarded as "twice" as important as the other two in scenario 2. In scenario 3 criterion B is considered clearly more important than two other ones; it is deemed "twice" as important as the two others. In scenario 4 the emphasis is on criterion $\mathrm{C}$ which is regarded as "twice" as important as criteria A and B. Table 2 summarizes the scenarios.

\begin{tabular}{|l|l|l|l|l|}
\cline { 2 - 5 } \multicolumn{1}{c|}{} & Scenario $1^{2)}$ & Scenario 2 & Scenario 3 & Scenario 4 \\
\hline CRITERION A & $0.33(0.0471)$ & $0.5(0.070)$ & $0.25(0.036)$ & $0.25(0.036)$ \\
\hline CRITERION B & $0.33(0.055)$ & $0.25(0.042)$ & $0.5(0.083)$ & $0.25(0.042)$ \\
\hline CRITERION C & $0.33(0.0825)$ & $0.25(0.0625)$ & $0.25(0.0625)$ & $0.5(0.125)$ \\
\hline \multicolumn{3}{|c|}{ Cardinal weights }
\end{tabular}

\begin{tabular}{|l|l|l|l|l|}
\cline { 2 - 5 } \multicolumn{1}{l|}{} & Scenario 1 & Scenario 2 & Scenario 3 & Scenario 4 \\
\hline CRITERION A & \multirow{2}{*}{$\mathrm{wA}=\mathrm{wB}=\mathrm{wC}$} & $\mathrm{wA}>\mathrm{wB}=\mathrm{wC}$ & $\mathrm{wA}=\mathrm{wC}<\mathrm{wB}$ & WA = wB $<\mathrm{wC}$ \\
\hline CRITERION B & & & \\
\hline CRITERION C & & & \\
\hline
\end{tabular}

Table 2. The scenarios for the numerical experiments.

In Table 2 the term in parentheses is the weight per sub-criteria of that particular criterion. The results from the study of Agostiano [1995] are reported in the following figures $^{3)}$.

\footnotetext{
${ }^{2)}$ In scenario 1 Agostiano [1995] reported equal weights for the 17 sub-criteria (i.e., 0.058) but obviously used the correct weights shown in the table.

${ }^{3)}$ In Figures 1a-1d a higher value is always better.
} 


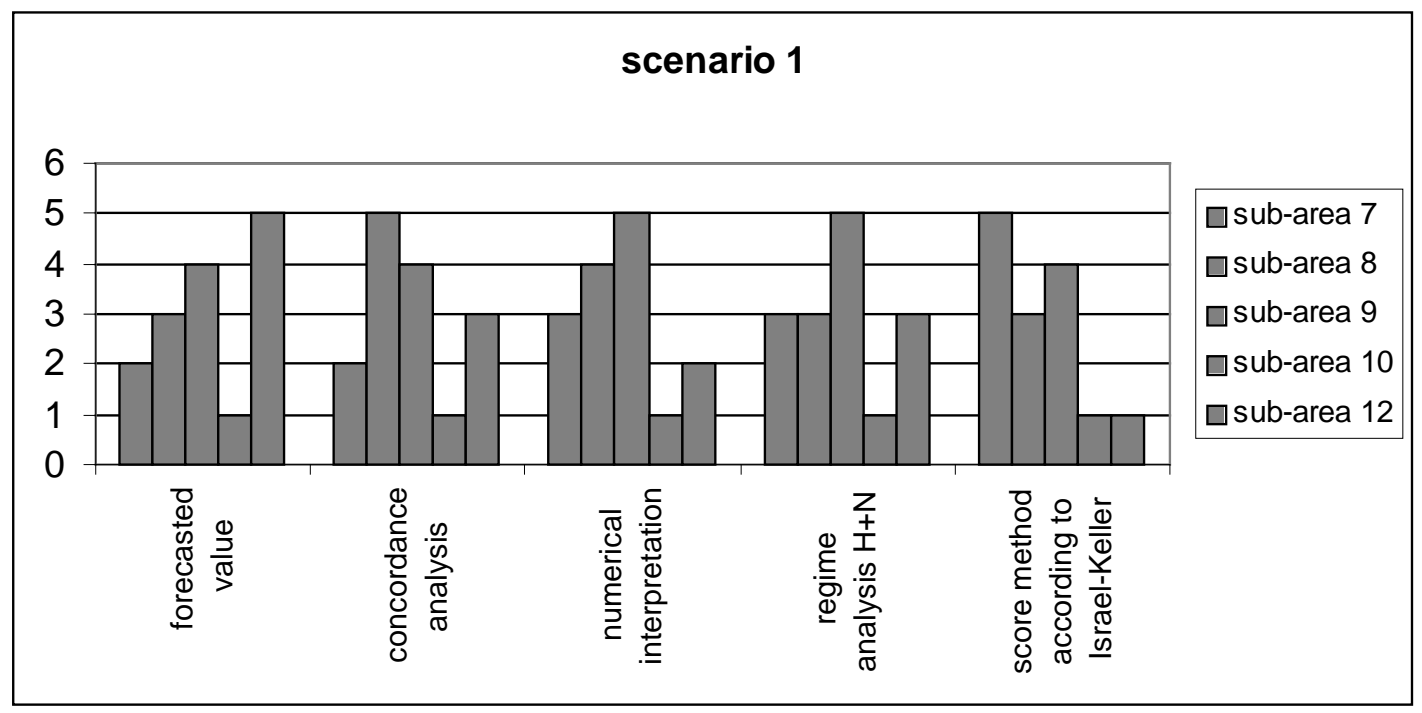

Figure 1a. Results from the five methods with weight scenario 1.

It is clear from Figure 1a that the five methods all result in different rank orders for the sub-areas when all criteria have equal weights (scenario 1). Except for sub-area 10 all other sub-areas are ranked first with at least one method, sub-area 9 with two methods. Sub-area 10 is ranked last with all methods. With the score method according to IsraelsKeller it ties this place with sub-area 12. It is interesting to notice that the method of forecasted value ranks sub-area 12 first, whereas the score method according to IsraelsKeller ranks it last. Regime analysis according to Hinloopen-Nijkamp results in three ties for the second place, which means that, in this case, it can only distinguish the best and the worst sub-areas.

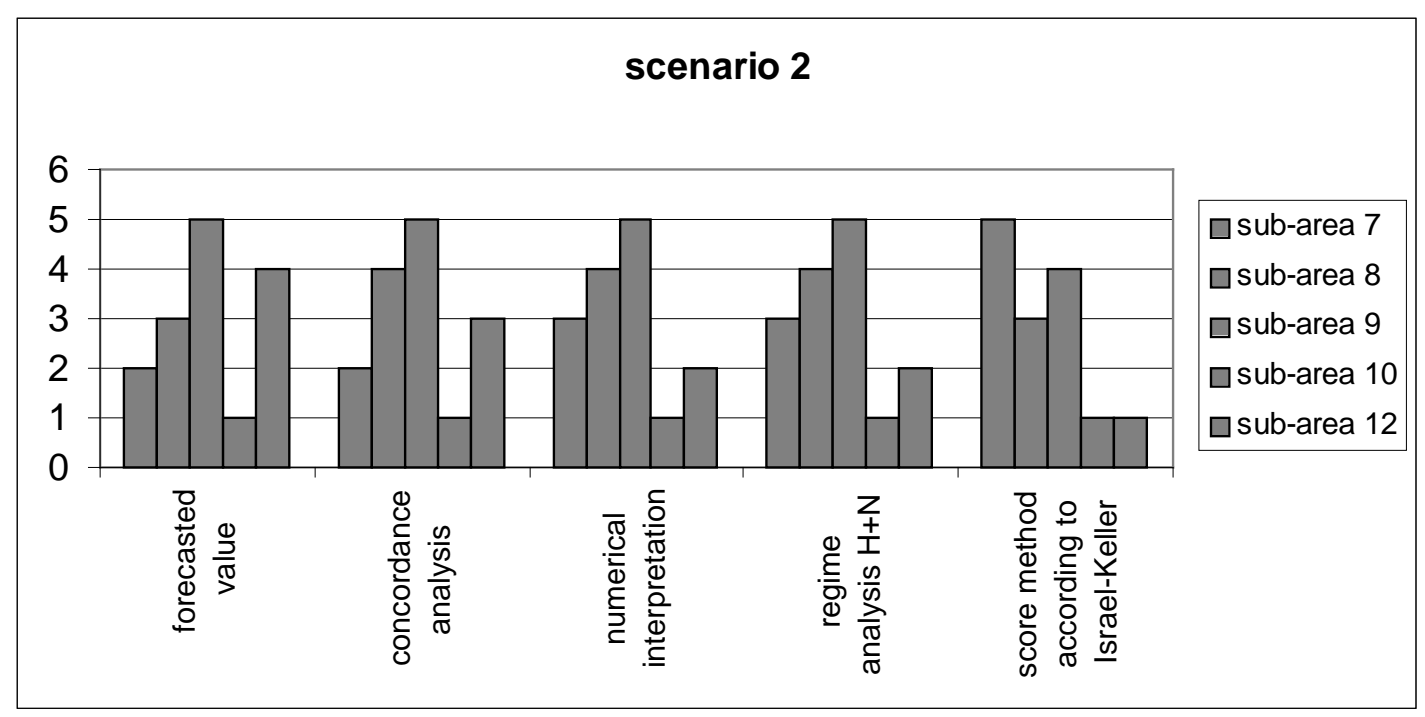

Figure 1b. Results from the five methods with weight scenario 2 . 
When more weight is put on criterion A (scenario 2), the methods of numerical interpretation and regime analysis according to Hinloopen-Nijkamp produce the same ranking for the sub-areas. This can be seen in Figure 1b. Moreover, the rank order with the method of numerical interpretation is the same as in scenario 1. This is also true for the score method according to Israels-Keller. Compared to scenario 1, the method of forecasted value and concordance analysis switch the sub-areas in the first and second places, sub-areas 9 and 12 and 8 and 9, respectively.

Sub-area 9 is ranked first with four methods and sub-area 7 with one method. In the latter case sub-area 9 is ranked second. As in scenario 1 sub-area 10 is ranked last with all methods and in the same way it ties this place with sub-area 12 when the score method according to Israels-Keller is used.

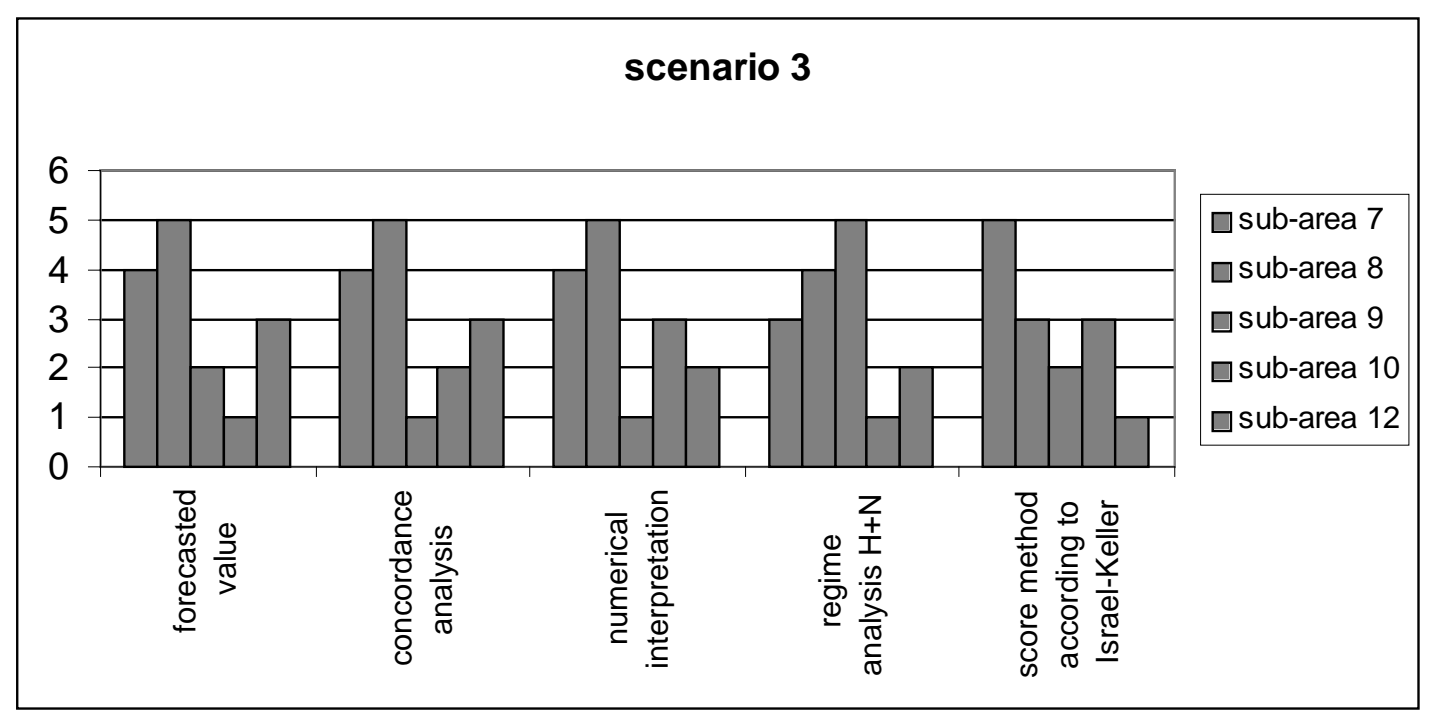

Figure 1c. Results from the five methods with weight scenario 3.

In scenario 3 with emphasis on criterion B (Figure 1c) the five methods result again in different rankings. However, regime analysis according to Hinloopen-Nijkamp generates the same rank order as in scenario 2 . This is also the same ranking as with the method of numerical interpretation in scenarios 1 and 2 .

In scenario 3, sub-area 8 is ranked first three times and sub-areas 7 and 9 each once. It is worth noticing that regime analysis according to Hinloopen-Nijkamp ranks sub-area 9 first whereas the methods of numerical interpretation and concordance analysis rank it last. 


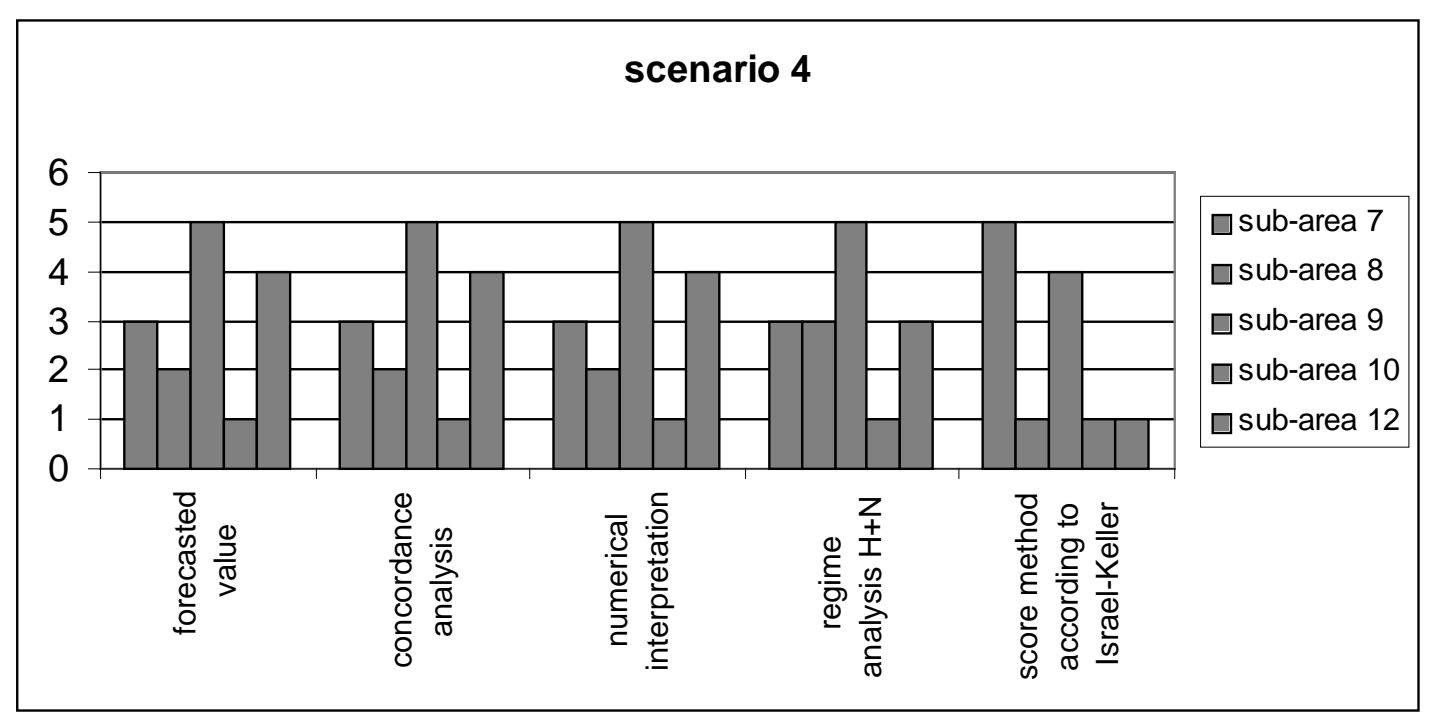

Figure 1d. Results from the five methods with weight scenario 4.

With more weight on criterion $\mathrm{C}$ the results seem more consistent than with emphasis on other criteria as Figure 1d shows. The same rank order is produced by the methods of forecasted value and numerical interpretation and concordance analysis. Regime analysis according to Hinloopen-Nijkamp ranks the sub-areas in the same order as in scenario 1 but this ranking has three ties for the second place.

In Figure 1d, sub-area 9 is ranked first in four cases and second once. Sub-area 7 is ranked first once. Sub-area 10 is always the last one with one tie with sub-areas 8 and 12 when the score method according to Israels-Keller is used.

To summarize the results, it is clear from Figures 1a-1d that the methods used do not usually produce the same rankings. In other words, it does make a big difference (i) which method one selects and (ii) what magnitude of weights one uses. But there are numerous arguments in MCDM against using importance weights as a means to elicit and represent decision maker's preference information (e.g., Steuer [1986, pp. 193-200], Korhonen and Wallenius [1989], Wierzbicki [1986]). This is established above as well where it is shown that it is possible to produce the same ranking although the weights differ. It is also not straightforward to argue that the greater the importance of a criterion, the larger the respective weight. Nakayama [1995] showed that there is no positive correlation between the weight and the value of the objective at the resulting solution. Therefore, we propose in Chapter 4 the use of an interactive method where one does not have to specify weights in advance.

\subsection{Critique of Agostiano's [1995] results}

Information in the evaluation matrix of Table 1 can be used to calculate a pairwise comparison matrix for each criterion. Because Agostiano [1995] used only ordinal data, we first calculate for all criteria unweighted pairwise comparison matrices. Assuming that all sub-criteria are considered equally important within the respective criterion we add the pairwise comparison matrices within each criterion. The results are given in 
Table 3. Each cell indicates how many times the alternative in row $\mathrm{i}$ is ranked higher than the alternative in column $\mathrm{j}$ within each criterion.

\begin{tabular}{||l||l|l|l|l|l||l|l|l|l|l|l|l|l|l|l||}
\hline \hline \multicolumn{1}{||c||}{} & \multicolumn{4}{c||}{$\begin{array}{c}\text { Criterion A } \\
\text { (7 sub-criteria) }\end{array}$} & \multicolumn{4}{c||}{$\begin{array}{c}\text { Criterion B } \\
\text { (6 sub-criteria) }\end{array}$} & \multicolumn{5}{c||}{$\begin{array}{c}\text { Criterion C } \\
\text { (4 sub-criteria) }\end{array}$} \\
\hline & 7 & 8 & 9 & 10 & 12 & 7 & 8 & 9 & 10 & 12 & 7 & 8 & 9 & 10 & 12 \\
\hline 7 & - & 4 & 3 & 4 & 3 & - & 3 & 3 & 2 & 3 & - & 2 & 1 & 4 & 1 \\
\hline 8 & 3 & - & 3 & 4 & 3 & 3 & - & 5 & 3 & 4 & 2 & - & 1 & 2 & 2 \\
\hline 9 & 4 & 3 & - & 3 & 3 & 2 & 1 & - & 2 & 2 & 3 & 3 & - & 3 & 2 \\
\hline 10 & 3 & 3 & 2 & - & 4 & 4 & 3 & 4 & - & 3 & 0 & 2 & 1 & - & 1 \\
\hline 12 & 4 & 3 & 3 & 3 & - & 1 & 2 & 3 & 2 & - & 3 & 2 & 2 & 3 & - \\
\hline \hline
\end{tabular}

Table 3. The unweighted pairwise comparison matrices.

From Table 3 it is easy to deduce the following "common sense" ranking rules:

1. sub-area 7 should always be ranked before sub-area 8 if they are on two consecutive places,

2. sub-area 8 should always be ranked before sub-area 10 if they are on two consecutive places,

3. sub-area 8 should always be ranked before sub-area 12 if they are on two consecutive places, and

4. sub-area 12 should always be ranked before sub-area 9 if they are on two consecutive places.

In other words, the first mentioned sub-area dominates the latter one irrespective of the possible weights for the criteria. In each case, the result is based on the fact that on two criteria the sub-areas are tied but on the third one the first mentioned is ranked before the latter one.

At least one of the "common sense" rules is violated for every method in the results for scenarios 3 and 4 in Section 3.3. For scenario 1 only score method according to IsraelsKeller does not break any of the four "common sense" rules. For scenario 2, concordance analysis and score method according to Israels-Keller obey the four rules above, the other three methods breach at least one of them.

In scenario 1 with equal weights for the criteria the score method according to IsraelsKeller places sub-area 7 ahead of sub-area 9. But if we look at the pairwise comparison matrices in Table 3, sub-area 9 should be ranked before sub-area 7 because (1) on criterion A sub-area 9 wins over sub-area 7 by 4-3 from 7 possibilities, (2) on criterion B sub-area 7 wins over sub-area 9 by 3-2 from 6 possibilities, and (3) on criterion C sub-area 9 wins over sub-area 7 by 3-1 from 4 possibilities.

On the other hand, in scenario 2 more weight is put on criterion A. In this case, concordance analysis ranks sub-area 9 before sub-area 8 . But these two sub-areas have the same number of pairwise winnings on criterion A. Since equal weight is to be put on criteria B and C, sub-area 8 should be ranked ahead of sub-area 9 because on criterion B sub-area 8 beats sub-area 9 by 5-1 out of 6 possibilities and on criterion $\mathrm{C}$ there is only a 
3-1 win for sub-area 8 out of 4 possibilities. Also score method according to IsraelsKeller ranks these two sub-areas incorrectly.

To summarize the result above, all five methods violate at least one of the pairwise ranking orders provided by the original evaluation matrix. This is true for all four scenarios.

\section{Rank Order Using Minimum Violation Princ iple}

We first calculate the pairwise comparison matrix for each criterion. We use equation (2.1) for the sub-criteria presenting value structure on an ordinal scale (sub-criteria 4A, $10 \mathrm{~B}$, and 11B), and for the rest of the sub-criteria we employ equation (2.2). Equation (2.1) defines a so-called unweighted pairwise matrix and equation (2.2) a weighted pairwise matrix. In the latter case, we assume that the value function is a linear monotonic function of the numerical values of the criterion. It is decreasing for the cost related sub-criteria (1A-3A), and increasing for the rest of the sub-criteria. All pairwise comparison matrices $\mathrm{C}^{\mathrm{j}}, \mathrm{j}=1,2, \ldots, 17$, are transformed into vector form as explained in equation (2.3). Each vector consists of $25(=5 \times 5)$ elements. We are looking for vector $\gamma$ (equation (2.7)) standing for a rank order, which simultaneously maximizes the correlation coefficient with each given vector $\mathbf{f}_{\mathrm{j}}, \mathrm{j}=1,2, \ldots, 17$. Unfortunately, none of the 120 possible rankings fulfills this requirement. On the other hand, all possible rank orders are nondominated (see, Definition 3). This is why any of those rank orders is a rational choice. But how do we find the best ranking?

There are a number of apparent advantages of employing an interactive multicriteria approach to help a DM to find his/her most preferred ranking from among the 120 possible nondominated ones. First, it is possible to explore the efficient frontier without determining the weights beforehand. (Actually, it is not possible to generate all nondominated solutions by using the weighted sums.) Second, by moving around the efficient frontier the DM is able to examine the mutual dependencies of the criteria. Third, the number of active criteria (that is, taken into account at the moment) can vary during the process as can the combination of active criteria. Fourth, the DM is free to apply any principle (e.g, the lexicographic one) he/she chooses to select the most preferred solution from among the proposed efficient ones. To summarize, the DM is in control of the process throughout the session. It is usually claimed that if the number of criteria is clearly less than ten, then the DM is able to evaluate the alternatives with all criteria simultaneously. However, 17 criteria are too many for this kind of approach. Therefore, he/she has to solve the problem in a stepwise manner. There are (at least) two reasonable approaches:

1. Alternatives are first evaluated and ranked within each criterion separately. Those three rank orders are then used as new criteria for finding the final rank order.

2. The information is first aggregated within each main criterion, and then the aggregated information is used for finding the final rank order.

In each approach, we unfortunately lose information. In the previous case, each new rank order does not any more include information about individual rankings. In the latter case, the aggregation process destroys some information. If weighted sums are used in aggregation, some potential rank orders will be eliminated from further consideration. We will consider these principles in the following section. 


\subsection{Cardinal information}

When we use all 17 sub-criteria VIMDA reveals that there are no dominated rankings. In other words, unless there is some preference information available, all rankings are both possible and rational choices. In this application, the DM is assumed to articulate his preference information over the main criteria. Therefore we have to use some principle to aggregate the lower-level information. Let us first consider the approach where the "best" rank order for each main criterion is searched.

When we consider sub-criteria below criterion A, all the 120 permutations of the rankings are nondominated. Hence, there is no rational principle to eliminate any of those possible rank orders. The DM's preference structure determines which one is chosen. Because we do not have this information available, for demonstrative purposes we assume that he/she would like to find a rank order, which maximizes the minimum correlation coefficient (see, problem (2.8)) between a rank order and the sub-criteria. The same principle is applied to criteria B and C. In these two cases, some alternatives can be eliminated, because, with criterion B, 102 alternatives are nondominated, and, with criterion C, only 31 alternatives are nondominated. The results are shown in Table 4.

\begin{tabular}{|l|l|l|l|l|l||}
\hline \multicolumn{1}{|c|}{} & \multicolumn{5}{|c|}{ Sub-area } \\
\cline { 2 - 6 } & 7 & 8 & 9 & 10 & 12 \\
\hline \hline Criterion A & 2 & 4 & 5 & 3 & 1 \\
\hline Criterion B & 4 & 2 & 5 & 3 & 1 \\
\hline Criterion C & 1 & 4 & 3 & 5 & 2 \\
\hline
\end{tabular}

Table 4. Rank orders of the sub-areas based on the maximum of minimum correlation between the sub-criteria and the rank order chosen. ${ }^{4}$

Assuming that "maximizing the minimum correlation"-principle corresponds to the DM's preferences we are able to reduce the 17 rank orders into three different orders and continue the analysis with these three new rankings from Table 4 . Because the DM's preference information is not available, we do not complete this analysis.

Another approach to reduce the number of criteria is to first aggregate all rank orders at the lower level to the level of the three main criteria by using some pre-specified principle. This can be done for example as follows: within each criterion, we calculate the average of the correlation coefficients; in other words, we assume that the subcriteria are considered equally important within each criterion. It means that our aggregated criterion is based on "average non-violation". This principle is used for comparative purposes, because it corresponds to the idea used by Agostiano [1995] in his analyses.

Table 5 below shows the rank orders of the sub-areas when we maximize the average of the correlation coefficients separately for each criterion.

\footnotetext{
${ }^{4)}$ In this and subsequent tables a higher value is always better.
} 


\begin{tabular}{|l||l|l|l|l|l|}
\hline \multicolumn{1}{|c|}{} & \multicolumn{5}{c|}{ Sub-area } \\
\cline { 2 - 6 } \multicolumn{1}{c|}{} & 7 & 8 & 9 & 10 & 12 \\
\hline \hline Criterion A & 5 & 1 & 2 & 3 & 4 \\
\hline Criterion B & 3 & 5 & 1 & 4 & 2 \\
\hline Criterion C & 3 & 2 & 5 & 1 & 4 \\
\hline
\end{tabular}

Table 5. Rank orders of the sub-areas based on "average non-violation" for each criterion separately.

In this way the problem is reduced into a new three-criteria one where each criterion is based on average non-violation. Using VIMDA software we can ascertain that there are 53 dominated alternatives and 67 nondominated ones. To choose the "most preferred solution" for each scenario, we use the weights for the aggregated criteria (in Table 2) as proposed by Agostiniano [1995] and add the results over the criteria. We then select for each scenario the ranking that has the highest weighted sum. The results appear in Table 6.

\begin{tabular}{|l||l|l|l|l|l||}
\hline \multicolumn{1}{|c||}{} & \multicolumn{5}{c|}{ Sub-area } \\
\cline { 2 - 7 } & 7 & 8 & 9 & 10 & 12 \\
\hline \hline Scenario 1 & 4 & 2 & 3 & 1 & 5 \\
\hline Scenario 2 & 4 & 2 & 3 & 1 & 5 \\
\hline Scenario 3 & 5 & 4 & 2 & 1 & 3 \\
\hline Scenario 4 & 3 & 2 & 5 & 1 & 4 \\
\hline
\end{tabular}

Table 6 . Rank orders of the sub-areas based on weighted "average minimum violation" for the three criteria.

It is interesting that in Table 6 scenarios 1 and 2 result in the same rank order. In other words, putting equal weight on the criteria produces the same ranking as putting more weight on criterion A than on the other two.

We would like to emphasize that we have aggregated the rankings over the three main criteria only for demonstration purposes. Our proposal is to use an interactive approach and enable the DM to a make free search in the nondominated set. In this way, he/she is free to choose any nondominated alternative as his/her most preferred solution.

\subsection{Ordinal information}

In order to be able to compare the results from our minimum violation principle to those of Agostiano [1995] we can only use ordinal information. Following the procedure described in the beginning of this chapter we first calculate unweighted pairwise comparison matrices for all sub-criteria, be they cardinal or ordinal. Then we aggregate over the sub-criteria by computing for each criterion an "average non-violation", and use the aggregated values in the final analysis. 
We first use VIMDA to determine the number of nondominated rankings. We find 95 dominated rankings, which, however, leaves 25 nondominated ones.

Table 7 below shows for each scenario in Table 2 the rank orders of the sub-areas based on ordinal data and the average minimum violation principle.

\begin{tabular}{|l||l|l|l|l|l||}
\hline \multicolumn{1}{|c||}{} & \multicolumn{5}{c|}{ Sub-area } \\
\cline { 2 - 6 } & 7 & 8 & 9 & 10 & 12 \\
\hline \hline Scenario 1 & 2 & 5 & 3 & 1 & 4 \\
\hline Scenario 2 & 2 & 5 & 3 & 1 & 4 \\
\hline Scenario 3 & 2 & 5 & 3 & 1 & 4 \\
\hline Scenario 4 & 2 & 4 & 5 & 1 & 3 \\
\hline
\end{tabular}

Table 7. Rank orders of the sub-areas based on weighted "average non-violation" for the three criteria.

It is interesting to note that the first three scenarios in Table 7 produce the same rank order, although they emphasize different criteria. None of the rankings in Table 7 equal the ones that Agostiano [1995] produced with the five methods shown in Figures 1a-1d. However, scenario 4 results in the same rank order as concordance analysis with scenario 2 (see Figure 1b). It will be shown later that the rank order in scenario 4 in Table 7 does not break any of the "common sense" rules as scenario 2 did with concordance analysis.

The rankings in Table 7 do not violate any of the four "common sense" ranking rules based on pairwise comparison matrices in Table 3 and introduced in Section 3.4. As regards scenario 1 with equal weights the justification for ranking sub-area 9 ahead of sub-area 7 can be found in Section 3.4. By examining the pairwise comparison matrices in Table 3 and bearing at the same time in mind that the criteria have equal weights it is easy to reach the conclusion that sub-area 7 should be ranked before sub-area 10 . On criterion A sub-area 7 wins over sub-area 10 by $4-3$ out of 7 possibilities. Sub-area 10 wins over sub-area 7 with 4-2 out of 6 possibilities on criterion B but loses on criterion C by $0-4$ out of 4 possibilities.

For scenario 2 we have more emphasis on criterion A and criteria $\mathrm{B}$ and $\mathrm{C}$ have equal weights. Also in this case sub-area 9 should be ranked before sub-area 7 in pairwise comparison. On criterion B sub-area 7 wins over sub-area 9 by 3-2 out of 6 possibilities but on criterion $C$ sub-area 9 wins over sub-area 7 by $4-0$ out of 4 possibilities. Moreover, for criterion A sub-area 9 wins over sub-area 7 by 4-3 out of 7 possibilities. Hence, sub-area 9 should be ranked before sub-area 7 . The reasoning why sub-area 7 should be ranked ahead of sub-area 10 can be deduced in a similar way. On criterion B sub-area 7 loses to sub-area 10 by 2-4 out of 6 possibilities but on criterion $C$ it wins with 4-0 out of 4 possibilities. In addition, sub-area 7 wins over sub-area 10 on criterion A by 4-3 out of 7 possibilities.

When criterion $\mathrm{B}$ is considered to have more weight, we deal with scenario 3 . Because in this case criteria $A$ and $C$ have the same weights sub-area 9 should be ranked in pairwise comparison before sub-area 7 with regard to these two criteria. Sub-area 9 wins over sub-area 7 with 4-3 out of 7 possibilities on criterion A and with 3-1 out of 4 
possibilities on criterion C. Sub-area 9 loses however to sub-area 7 by 2-3 out of 6 possibilities on criterion B. But only by putting an extremely high weight on this criterion is it possible to rank sub-area 7 before sub-area 9. As to sub-areas 7 and 10, sub-area 7 is so strong in pairwise comparison on criterion A (win with 4-3 out of 7 possibilities) and on criterion $C$ (win with 4-0 out of 4 possibilities) that its defeat on criterion B (loss with 2-4 out of 6 possibilities) does not affect the rank order unless criterion $\mathrm{B}$ is regarded as remarkably more important.

In scenario 4 we emphasize criterion C. Sub-areas 9 and 8 are equally strong on criterion A. Sub-area 8 is very much stronger on criterion B. It wins over sub-area 9 by 5-1 out of 6 possibilities. But, on criterion $\mathrm{C}$ that is regarded as the most important one in this case, sub-area 9 clearly comes first. It wins over sub-area 8 by 3-1 out of 4 possibilities. In order to rank sub-area 8 ahead of sub-area 9 the weight for criterion B should be higher than it presently is. Therefore, we conclude that sub-area 9 should be ranked before sub-area 8 . Sub-area 12 wins over sub-area 7 on criterion A by 4-3 out of 7 possibilities. It loses on criterion B with 1-3 out of 6 possibilities. But on criterion $\mathrm{C}$ it again wins by 3-1 out of 4 possibilities. Therefore, sub-area 12 is so much stronger in the latter case that it should be ranked ahead of sub-area 7. For sub-areas 7 and 10 it is clear that the first one should be come before the latter one. On the two criteria with equal weights, A and B, sub-area 7 wins on the first one by 4-3 out of 7 possibilities and loses on the second one by 2-4 out of 6 possibilities. For criterion $C$, which is regarded as more important than the two others, sub-area 7 is always ranked ahead of sub-area 10; in other words, it wins over sub-area 10 by $4-0$ out of 4 possibilities. Consequently, sub-area 7 should be ranked ahead of sub-area 10 .

Based on the analyses above we conclude that the results in Table 7 are consistent with those of pairwise comparison. In conclusion it is worth emphasizing that they do not break any of the "common sense" ranking rules from Section 3.4.

\section{Concluding remarks}

In this paper, we have studied a problem of urban rehabilitation, which involves five sub-areas. Each of these represents a possible rehabilitation project. The sub-areas are characterized with seventeen criteria, which are grouped into three main criteria. On each criterion the sub-areas are presented either on an ordinal or on a cardinal scale. The purpose is to find a rank order for these sub-areas, which simultaneously determines where to start the rehabilitation process.

The problem was previously considered by Agostiano [1995], who used five different methods. Agostiano reported an expected result: different methods result in different rank orders. However, he did not propose any guidelines for how a decision maker might choose the best method. We have proposed the use of the "minimum violation" principle for comparison of different methods. We have also proposed a ranking method that is based on this principle. The purpose is to solve a multiple objective integer linear programming model. Each objective function describes the (standardized) number of pairwise violated rank orders between each original rank order and the rank order to be searched. The method is interactive and it allows the decision maker to freely search possible (non-dominated) solutions and to choose any of these as his/her most preferred one. This approach is able to deal with both ordinal and cardinal information. 
We have demonstrated that our approach gives results that are consistent with some "common sense" rules. None of the five methods included in Agostiano's [1995] experiment fulfilled all these "common sense" rules.

\section{REFERENCES}

Agostiano, M. (1995), La valutazione dei piani di recupero di centri storici: un'applicazione di Multicriteria Analysis e Community Impact Evaluation per i Sassi di Matera. Degree Thesis, Politecnico di Bari, Dipartimento di Architettura e Urbanistica,.tutor Borri Dino

Albers, L.H. (1989), "A classification of historical farmgardens in the north of the Netherlands", in A. Barbanente (Ed.), (1992), La valutazione nella pianificazione urbana e regionale, vol.I, n.8, IRIS, CNR, Bari.

Albers, L., and Nijkamp, P. (1989), "Analisi multidimensionale per la valutazione dei piani e dei progetti. Come adeguare il giusto metodo al giusto problema" in A. Barbanente (Ed.), (1992), La valutazione nella pianificazione urbana e regionale, vol.II, n.8, IRIS, CNR, Bari.

Bizzarro, F., and Ferretti, F. (1997), "La scelta della destinazione d'uso per la riqualificazione di un'insula nel centro storico di Napoli", in L. Fusco Girard and P. Nijkamp, Le valutazioni per lo sviluppo sostenibile della città e del territorio, Franco Angeli, Milano.

Bowman, V. and Colantoni, C. (1972), "Majority Rule under Transitivity Constraints", Management Science 19, 1029-1041.

Daniels, H. (1943-46), "The Relation between Measures of Correlation in the Universe of Sample Permutations", Biometrika XXXIII, 129-135.

De Toro, P. (1997), "La valutazione di tre proposte alternative di sistemazione dell'area di Coroglio a Napoli" in L. Fusco Girard and P. Nijkamp, Le valutazioni per lo sviluppo sostenibile della città e del territorio, Franco Angeli, Milano.

Delft, A., and Nijkamp, P. (1977), Multicriteria Analysis and Regional Planning, The Hague.

Fusco Girard, L. (1987), Risorse architettoniche ed ambientali, valutazioni e strategie di conservazione, Franco Angeli, Milano.

Giordano, G. (1997), "La valutazione di alternative nel recupero del Boeo Medievale di Maratea", in L. Fusco Girard and P. Nijkamp, Le valutazioni per lo sviluppo sostenibile della città e del territorio, Franco Angeli, Milano.

Hinloopen, E., Nijkamp, P., and Rietveld, P. (1983), "The regime method: A new multicriteria technique", in: P. Hansen (ed.), Essays and Surveys on Multiple Criteria Decision Making, Springer, 146-155.

Israels, A. Z. and Keller, W. J. (1986), "Multicriteria Analyse voor Ordinale Data", Kwantitatieve Methoden 7, 49-74. 
Korhonen, P. (1986), "A Hierarchical Interactive Method for Ranking Alternatives with Multiple Qualitative Criteria", European Journal of Operational Research 24, 265-276.

Korhonen, P. (1988), "A Visual Reference Direction Approach to Solving Discrete Multiple Criteria Problems", European Journal of Operational Research 34, 152-159.

Korhonen, P. and Soismaa, M. (1981), "An Interactive Multiple Criteria Approach to Ranking Alternatives", Journal of the Operational Research Society 32, 577-585.

Korhonen, P. and Wallenius, J. (1989), "A Careful Look at Efficiency and Utility in Multiple Criteria Decision Making: A Tutorial", Asia-Pacific Journal of Operational Research 6, 46-62.

Nakayma, H. (1995), "Aspiration Level Approach to Interactive Multi-Objective Programming and its Applications" in P. M. Pardalos, Y. Siskos, and C. Zopounidis (eds.), Advances in Multicriteria Analysis, Kluwer Academic Publishers, 147-174.

Nijkamp, P. (1975), "A Multicriteria Analysis for Project Evaluation", Papers of Regional Science Association 35.

Nijkamp, P. (1988), "Multidimensional Evaluation of Monuments", Environment and Planning 16.

Nijkamp, P., Rietveld, P., and Voogd, H. (1990), Multicriteria Evaluation in Physical Planning, North-Holland, Amsterdam.

Rietveld, P. (1980), Multiple Objective Decision Methods and Regional Planning, North-Holland, Amsterdam.

Roy, B. (1968), "Classement et Choix en Présece de Points de Vue Multiples (la Méthode ELECTRE", R.I.R.O. 2, 57-75.

Roy, B. (1972), "Décision avec Criteres Multiples", Metra 11, 121-151.

Steuer, R.E. (1986), Multiple Criteria Optimization: Theory, Computation, and Application, Wiley, New York.

Voogd, H. (1982), "Multicriteria Evaluation with Mixed Qualitative and Quantitative Data", Environment and Planning 9.

Voogd, H. (1983), Multicriteria Evaluation for Urban and Regional Planning, Pion, London.

Wierzbicki, A. (1986), "On the completeness and constructiveness of parametric characterizations to vector optimization problems", OR Spectrum 8, 73-87. 